\title{
Association between blood lactate and acid-base status and mortality in ventilated babies
}

\author{
S A Deshpande, M P Ward Platt
}

\begin{abstract}
Aim-To investigate the relation between common acid-base parameters and blood lactate concentrations and their prognostic importance in sick, ventilated neonates.

Methods-Two hundred and seventy eight serial simultaneous measurements of arterial acid-base status and blood lactate concentrations were carried out in 75 mechanically ventilated neonates with indwelling arterial catheters (gestational age and birthweight, median (range) - 29 (23-40) weeks, and 1340 (550-4080) $\mathrm{g}$, respectively).
\end{abstract}

Results-There were no correlations between arterial blood lactate and $\mathrm{pH}$ and base excess within subjects $(r=0.07$ and $r=$ -0.06 , respectively) and only weakly positive but clinically irrelevant positive correlations between subjects $(r=0.28$ and $\mathbf{r}=0.27$ ) in this group. Even in those infants who had not received any bicarbonate before their initial measurements $(n=48)$, there were no correlations between initial blood lactate concentrations and $\mathbf{p H}$ $(r=0.27)$, base excess $(r=0.17)$, or serum bicarbonate concentrations $(r=-0.18)$. There was no relation between peak lactate concentration (PLC) and base excess $(r=0.16)$, and only a weak correlation between peak lactate concentration (PLC) and $\mathrm{pH}(\mathrm{r}=0.28)$. Negative base excess was an insensitive indicator of raised lactate concentrations. Only two out of $33(6 \%)$ instances of hyperlactataemia (lactate $>2.5 \mathrm{mmol} / \mathrm{l}$ ) would have been identified with a base excess $<-10 \mathrm{mmol} / 1$ as a cutoff. Lower cutoff values of base excess or $\mathrm{pH}$ performed no better. Raised lactate concentrations were associated with increased mortality at all levels. While six of $53(11 \%)$ infants with a PLC $<2.5 \mathrm{mmol} / 1$ died, this proportion increased to four of $15(27 \%)$ with a PLC between 2.5-5.0 mmol/1, and four of seven (57\%) with a PLC $>5.0 \mathrm{mmol} / 1$. Infants showing little rise or a substantial fall in blood lactate fared better than those with persistently raised values. A clinically important increase in blood lactate preceded the development of clinical markers of deterioration and complications in six infants.

Conclusions-Contrary to popular belief, pH or base excess cannot be used as proxy measures for blood lactate concentration, and independent measurements of the latter are needed. Blood lactate concentrations may provide an early warning signal and important prognostic information in ill, ventilated neonates. In this regard, serial measurements of blood lactate are more useful than a single value. (Arch Dis Child 1997;76:F15-F20)

Keywords: blood lactate; base excess; pH; neonatal intensive care.

Hypoxia and hypotension are common in sick neonates, particularly preterm infants receiving intensive care. Monitoring of tissue perfusion is essential for early recognition of circulatory failure, institution of appropriate treatment, and in assessing response. Changes in heart rate, blood pressure, skin perfusion and urine output, which are generally used to detect tissue malperfusion in this age group, are insensitive markers as clinically relevant cellular hypoxia and malperfusion may be present well before clinically apparent changes in cardiovascular responses are seen. The size of these infants has generally precluded the use of more invasive haemodynamic monitoring, such as pulmonary artery thermodilution catheters to measure cardiac output, mixed venous oxygen saturations, and oxygen delivery.

Measurement of blood lactate concentrations is another tool used in critically ill adults for assessing tissue perfusion. With a critical reduction in oxygen and substrate delivery, aerobic metabolism through the Kreb's cycle cannot be sustained, and tissues increasingly need anaerobic metabolism to meet their energy needs. This, in turn, results in increased production and accumulation of blood lactate. Studies in experimental animals and critically ill adults have shown that the degree of increase in blood lactate is proportional to the severity of oxygen deficiency and the decrease in oxygen delivery. ${ }^{12}$ Blood lactate concentrations in critically ill and injured adult patients can be used to detect tissue hypoxia at an early stage, assess illness severity, and predict outcome..$^{3-6}$ Serial measurements of blood lactate concentrations are more valuable than a single measurement, not only in providing a more precise assessment of prognosis, but also in evaluating response to treatment.

Despite this volume of information on the value of blood lactate measurement in ill adults, information on its importance in critically ill neonates is limited. Hyperlactataemia is associated with increased mortality in premature infants with respiratory distress syndrome, ${ }^{89}$ and in term infants with severe 
respiratory failure receiving extracorporeal membrane oxygenation (ECMO).$^{10}$ Increased lactate concentrations in preterm infants have also been reported to be early indicators of sepsis $^{11}$ and the need for blood transfusions. ${ }^{12} 13$ In clinical practice, presence of acidosis, and in particular, negative base excess ("base deficit") is commonly assumed to reflect impaired tissue perfusion and hyperlactataemia. However, we could find no published evidence to support this, and studies of adult patients have shown either no correlations between acid-base status and blood lactate, ${ }^{14} 15$ or significant but clinically unimportant correlations between them. ${ }^{16}$ Despite this, $\mathrm{pH}$ and base excess continue to be used as proxy measures for tissue perfusion in neonates, and both therapeutic interventions and illness severity scores are based on these measurements. ${ }^{17}$

This study examines the relation between $\mathrm{pH}$ and negative base excess with arterial blood lactate concentrations in a population of mechanically ventilated neonates.

\section{Methods}

Ethical approval for the study was granted by the Joint Ethics Committee of Newcastle Health Authority and the University of Newcastle upon Tyne.

We prospectively studied serial arterial acidbase status and blood lactate concentrations in 75 consecutive sick, ventilated neonates with indwelling arterial lines, who had been admitted to the neonatal intensive care units in Newcastle upon Tyne. Their median (range) gestational age was 29 (23-40) weeks and birthweight 1340 (550-4080) g. There were 41 boys and 34 girls. Twenty four infants were less than 28 weeks of gestational age while 18 babies weighed less than $1000 \mathrm{~g}$ at birth. Forty six babies needed intermittent positive pressure ventilation for resuscitation. Surfactant deficiency respiratory distress syndrome (RDS) was the commonest reason for ventilation $(n=60)$, followed by persistent pulmonary hypertension $(n=4)$, prematurity $(n=3)$, group B streptococcal pneumonia $(n=3)$, congenital malformations of the respiratory tract $(n=3)$ and recurrent apnoea and asphyxia (one each). All these infants received intravenous $10 \%$ glucose infusions, with the total fluid intake varying between $100-165 \mathrm{ml} / \mathrm{kg} /$ day. None of these infants received intravenous amino acid solution, lipid emulsion, or lactate containing fluids during the study period. No attempt was made to control the administration of volume expanders such as $4.5 \%$ human albumin and/or packed cell transfusions, inotropes, or alkali. The results of blood lactate measurement were not available to the clinical team during the study period.

Blood samples were collected from indwelling arterial catheters at approximately 12 hourly intervals for a maximum of five serial measurements per baby. The study was terminated early in the event of death or removal of the arterial catheter either due to complications or a substantial improvement in the infant's status. Blood gas determinations were carried out using AVL-995 (AVL Medical Instruments UK Ltd, Staffs) and Corning 178 (Ciba Corning Diagnostics Ltd, Essex) pH/blood gas analysers.

At each of these blood gas determinations, $40 \mu \mathrm{l}$ of the residual blood from the syringe were collected in a volumetric microcapillary tube containing heparin and immediately placed into a tube containing $200 \mu \mathrm{l}$ of chilled $5 \%$ perchloric acid. The samples were immediately separated and the supernatant fluid frozen at $-80^{\circ} \mathrm{C}$ pending analysis. Whole blood lactate concentrations were measured using a microenzymatic lactate dehydrogenase method with a Cobas Bio fast centrifugal analyser. ${ }^{18}$ The intra-assay and interassay standard deviations (coefficients of variation) for the lactate assay were $0.016(5.6 \%)$ and $0.026(7.7 \%)$, respectively. Samples from each subject were run in a single assay, thereby avoiding interbatch variation in an individual subject.

For the purposes of this study, hyperlactataemia was defined as an arterial blood lactate concentration of greater than $2.5 \mathrm{mmol} / \mathrm{l}$. Data were analysed using the MINITAB statistical package (Clecom, Birmingham). The relation between acid-base parameters $(\mathrm{pH}$ and base excess) and blood lactate concentrations was studied between subjects (with the mean blood lactate concentrations for each subject in both groups) and within subjects (by calculating the residuals for each measurement from the mean for that subject). Peak lactate concentrations between various groups were compared using Student's $t$ test after logarithmic transformation. Pearson's correlation coefficients were used to define the relation between acid-base parameters and blood lactate concentrations. As the base excess values were not normally distributed and they could not be log transformed, Spearman's rank test was used to evaluate their relation with blood lactate values. Fisher's exact test was used to study the association between acid-base parameters and blood lactate with outcome.

\section{Results}

A total of 278 simultaneous measurements of arterial acid-base parameters and blood lactate were obtained from these 75 neonates. The initial sample was obtained at a median (range) age of 20 (1-68) hours after birth. In all but seven infants the first study sample was obtained more than eight hours after birth, reflecting the high proportion of outborn babies being transferred into the units.

There was no correlation between common acid-base parameters such as $\mathrm{pH}$, base excess, bicarbonate concentrations and blood lactate concentrations (fig 1). Neither $\mathrm{pH}$ nor base excess showed any association with blood lactate concentrations within subjects $(r=0.075$ and $r=-0.063$ respectively; $P>0.2$ ). There was a weakly positive intersubjects correlation between blood lactate and $\mathrm{pH} \quad(\mathrm{r}=0.284$; $\mathrm{P}<0.02)$ and base excess $(\mathrm{r}=0.272 ; \mathrm{P}=0.04)$. This is, however, contrary to the expected negative correlation between these acid-base parameters and blood lactate concentrations. There was a significant but probably clinically 


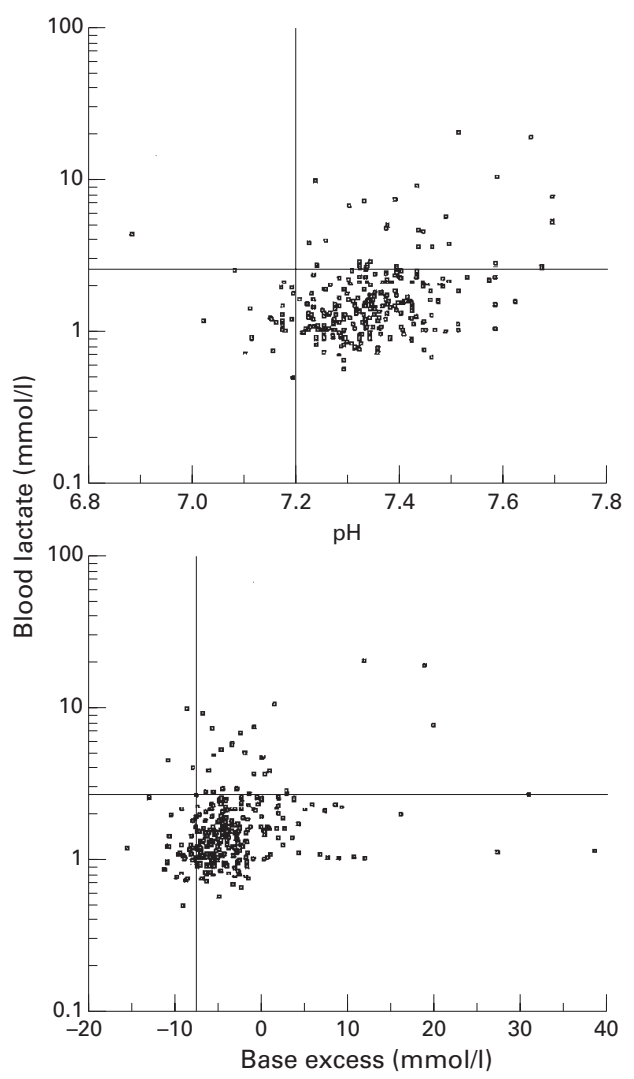

Figure 1 Correlations of arterial blood $\mathrm{pH}$ and base excess with blood lactate concentrations

irrelevant weakly positive correlation between PLC and concurrent $\mathrm{pH}(\mathrm{r}=0.279 ; \mathrm{P}<0.05)$. We could not find any correlation between PLC and base excess $(r=0.162 ; \mathrm{P}>0.2)$.

Forty eight infants did not receive any alkali treatment before their first study sample was taken. Even in these infants, there were no correlations between initial blood lactate concentrations and $\mathrm{pH} \quad(\mathrm{r}=0.269 ; \mathrm{P}>0.05)$, base excess $(r=0.169 ; P>0.2)$, or serum bicarbonate concentrations $(r=-0.179 ; \mathrm{P}>0.2)$. In the remaining 27 infants in whom initial acid-base and lactate measurements were obtained after administration of bicarbonate, there were positive correlations between blood lactate with base excess and bicarbonate concentrations $(\mathrm{r}=0.511 ; \mathrm{P}<0.02$ and $\mathrm{r}=0.405 ; \mathrm{P}<0.05$, re-
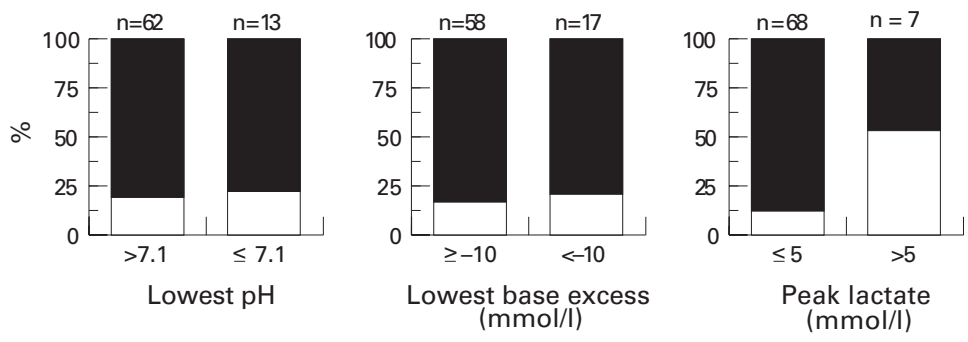

Survivor

Non-survivors

Figure 2 Inpatient mortality in relation to the lowest $p H\left(7.1 ; P^{\star}=0.716\right)$, worst base excess $\left(-10 \mathrm{mmol} / \mathrm{l} ; P^{\star}=0.734\right)$, and peak blood lactate concentrations (5 mmol/l; $\left.P^{\star}=0.026\right){ }^{\star}$ Fisher's exact test spectively) but none with $\mathrm{pH} \quad(\mathrm{r}=0.251$; $\mathrm{P}>0.2$ ).

Twenty two $(29 \%)$ neonates had hyperlactataemia, defined as arterial blood lactate concentration in excess of $2.5 \mathrm{mmol} / \mathrm{l}$. Neither $\mathrm{pH}$ nor base excess were sensitive indicators of hyperlactataemia. With a base excess less than $-10 \mathrm{mmol} / 1$ as a cutoff, only two of $33(6 \%)$ instances of hyperlactataemia would have been identified. Even changing this cutoff value to -8 or $-5 \mathrm{mmol} / \mathrm{l}$ did not significantly improve the detection of hyperlactataemia. Low $\mathrm{pH}$ performed no better. Using a $\mathrm{pH} \leqslant 7.20$ as a criterion of significant acidosis, 30 of $33(91 \%)$ of instances of hyperlactataemia would not have been identified. In all three instances of blood lactate concentrations $\geqslant 10 \mathrm{mmol} / 1$, neither $\mathrm{pH}$ nor base excess were abnormal.

Fifteen $(20 \%)$ infants died during their hospital stay. Raised blood lactate concentrations but neither the lowest $\mathrm{pH}$ nor the worst base excess were associated with increased inpatient mortality (fig 2 ). The inpatient mortality was $11 \%(6 / 53)$ with PLC $\leqslant 2.5 \mathrm{mmol} / 1$ and increased with increasing PLC, being $27 \%$ (4/15) when PLC was 2.501-5.0 $\mathrm{mmol} / 1$, and $57 \%$ (4/7) when PLC $>5.0 \mathrm{mmol} / \mathrm{l}$; both infants with PLC $\geqslant 10 \mathrm{mmol} / 1$ died in the early neonatal period.

Not all infants with hyperlactataemia died. Serial measurements of blood lactate were available in 66 infants. Of 49 who never became hyperlactataemic, only four infants died. Of 12 infants who had persistently high or continuously rising blood lactate, nine died. Infants showing a consistent decrease or only a modest increase in blood lactate concentrations during the study period generally did well. This was true even of those infants with initial hyperlactataemia. The subsequent change in blood lactate concentration, once it had exceeded a value of $2.5 \mathrm{mmol} / 1$, was also useful in differentiating survivors from nonsurvivors. Having reached a value of $>2.5$ $\mathrm{mmol} / \mathrm{l}$, the subsequent median (range) per cent change in survivors was $-44.73 \%(-32.2$ to $-65.1)$ while that in non-survivors was $-7.22 \%$ $(-17.5$ to +0.9$)$, $(\mathrm{P}=0.008 ; 95 \% \mathrm{CI}=30.06-$ 54.23) (fig 3). In six infants a rise in blood lactate concentrations preceded the clinical development of a complication such as hypotension $(n=4)$, necrotising enterocolitis $(n=1)$, or renal failure $(n=1)$.

Six infants had evidence of persistent pulmonary hypertension. All these infants were treated with a similar protocol, consisting of volume expansion, inotropic support with dobutamine, pulmonary arterial vasodilatation with tolazoline, hyperventilation and alkalisation. None of these infants received ECMO support, although three of them entered an ongoing trial of ECMO. Blood lactate concentrations in the non-survivors from this group were greater than $5.0 \mathrm{mmol} / \mathrm{l}$ and seemed to be useful indicators of outcome (table 1).

\section{Discussion}

This was an observational study on a selected population of infants. Only those ventilated infants with indwelling arterial lines were cho- 


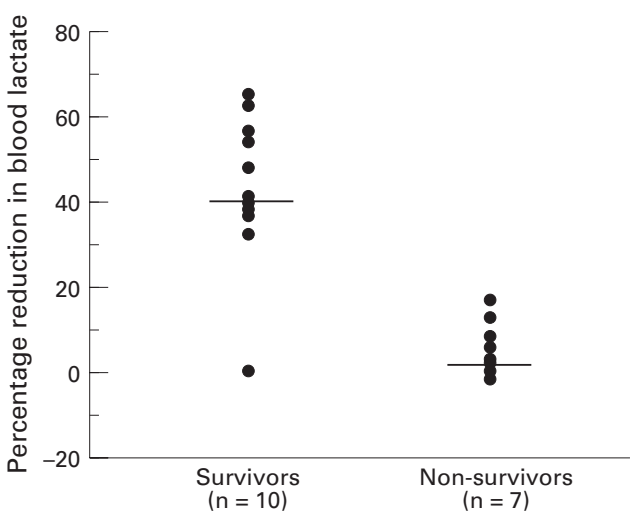

Figure 3 Per cent reduction in blood lactate concentrations in survivors and non-survivors after becoming hyperlactataemic (blood lactate $>2.5 \mathrm{mmol} / \mathrm{l}$ ) Median value for each group shown. Mann-Whitney U test showed that the two groups were significantly different from each other $(P=0.008)$.

sen, as the volume of blood needed for measurement of lactate $(40 \mu \mathrm{l})$ is generally present as residue in the syringe after blood gas determination. Thus there was no penalty to the baby in terms of extra blood loss for the study. Moreover, although good correlations have been shown between arterial blood lactate and mixed, central, and peripheral venous blood lactate concentrations, ${ }^{19-21}$ data on the use of capillary blood lactate as an alternative to arterial blood lactate are relatively sparse. Thus we chose not to include ventilated infants who did not have an indwelling arterial catheter. For obvious ethical reasons, it was not possible to define the normal ranges of arterial blood lactate concentrations in healthy infants. Previous studies of blood lactate concentrations in the umbilical arterial blood of healthy term and preterm infants show that most values are less than $2.5 \mathrm{mmol} / 1$ beyond the first six hours after birth. ${ }^{22}$ Moreover, blood lactate concentrations above $2 \mathrm{mmol} / \mathrm{l}$ are unusual in normal adults as well as nonhypotensive general ward patients ${ }^{24}$; concentrations below this are generally considered normal in critically ill adults. $^{25}$ A striking increase in mortality was shown in critically ill adults as blood lactate concentration increased $>2.5 \mathrm{mmol} / 1 .{ }^{26}$ Thus it seems reasonable to use blood lactate concentrations greater than 2.5 $\mathrm{mmol} / \mathrm{l}$ to define hyperlactataemia in neonates. We did not control the administration of alkalinising agents, volume expanders, and inotropes in this study. However, these interventions were not influenced by the blood lactate concentrations because these were unknown to the clinicians treating these infants.

Presence of metabolic acidosis (negative base excess) is often assumed to reflect inadequate tissue perfusion and oxygenation. The main purpose of our study was to examine the relation between common acid-base parameters and blood lactate concentrations, another indicator of tissue perfusion, in critically ill neonates. Tissue perfusion and oxygenation is a complex process mediated through a balance between oxygen delivery and consumption. Under physiological conditions, oxygen consumption is effected mainly through changes in the oxygen extraction. When oxygen extraction reaches the maximal value, oxygen delivery becomes critical in sustaining aerobic metabolism. Further reductions in oxygen delivery result in cellular oxygen deficit, and tissues switch to anaerobic glycolysis to meet their energy needs with increased production of lactate. Liver and, to a lesser extent, kidneys metabolise lactate through both oxidative and non-oxidative (mainly, gluconeogenesis) routes. Accumulation of blood lactate, therefore, occurs when increased production overwhelms the clearance mechanisms of this ion. Hyperlactataemia in circulatory failure is thus a rather late phenomenon that happens when oxygen extraction is near maximal and critical tissue hypoxia has occurred. In this respect changes in oxygen extraction and delivery are more sensitive markers of tissue hypoperfusion. However, these require measurement of parameters such as mixed venous oxygen saturation and cardiac output which are technically difficult in small sick neonates, and, therefore, markers of tissue hypoperfusion such as lactate have to be relied on in this population.

It has been suggested that in acute lactic acidosis the blood lactate concentration and base excess correlate well. ${ }^{27}$ In our study, however, we could not show any clinically relevant relation between common acid-base parameters, such as $\mathrm{pH}$ and negative base excess, and arterial blood lactate concentrations. These results agree with the observations in adults. ${ }^{141528}$ Cheung and others also could not find any significant linear relation between plasma lactate and arterial $\mathrm{pH}$ or serum bicarbonate values in infants referred for ECMO.$^{10}$ This is an important finding for two reasons. First, the presence of negative base excess, which is generally relied on to detect inadequate tissue perfusion, cannot be used as a proxy for blood lactate

Table 1 Ventilatory and oxygenation indices and blood lactate concentrations and in infants with persistent pulmonary hypertension

\begin{tabular}{|c|c|c|c|c|c|c|c|}
\hline $\begin{array}{l}\text { Case } \\
\text { No }\end{array}$ & Underlying disease & $\begin{array}{l}\text { Gestation } \\
\text { (weeks) }\end{array}$ & $\begin{array}{l}\text { Birthweight } \\
(g)\end{array}$ & $\begin{array}{l}\text { Mean airway } \\
\text { pressure (cms } \\
\left.\mathrm{H}_{2} \mathrm{O}\right)\end{array}$ & $\begin{array}{l}\text { Oxygenation } \\
\text { index }\end{array}$ & $\begin{array}{l}\text { Peak lactate } \\
\text { concentration } \\
\text { (mmol/l) }\end{array}$ & Outcome \\
\hline 1 & $\begin{array}{l}\text { Meconium aspiration } \\
\text { syndrome }\end{array}$ & 39 & 3425 & 27 & 90 & 2.74 & Recovered \\
\hline 2 & RDS & 36 & 3460 & 20 & 44 & 1.97 & Recovered \\
\hline 3 & RDS & 34 & 2211 & 25 & 75 & 20.0 & Died \\
\hline 4 & $\begin{array}{l}\text { Meconium aspiration } \\
\text { syndrome }\end{array}$ & 40 & 4080 & 23 & 58 & 2.27 & Recovered \\
\hline 5 & Diaphragmatic hernia & 38 & 3340 & 26 & 140 & 6.67 & Died \\
\hline 6 & Persistent fetal circulation & 38 & 3800 & 23 & 50 & 1.76 & Recovered \\
\hline
\end{tabular}


concentrations. Hence studies of interventions designed to improve tissue perfusion, such as the use of volume expanders or comparative trials of various inotropic agents, should include measurement of blood lactate as a marker of tissue perfusion. Second, although many babies in our study had their negative base excess corrected by the administration of alkali, their blood lactate continued to be high or rose. Thus correction of negative base excess may actually mask the continuing tissue malperfusion, and reliance on this parameter alone may provide a false reassurance of cellular wellbeing.

This lack of a relation between blood lactate and base excess may be due to several possible reasons. In most instances of hyperlactataemia, the arterial acid-base parameters were within normal range. Normalisation of $\mathrm{pH}$ through the administration of alkali is possibly the most important factor. However, this discrepancy between blood lactate concentrations and arterial $\mathrm{pH}$, base excess, and bicarbonate concentrations was also seen in infants prior to receiving any bicarbonate. There was a significant positive correlation of blood lactate with serum bicarbonate concentrations among infants who had received bicarbonate before their first study measurement. Alkalosis, particularly respiratory in origin, stimulates the rate limiting enzyme of glycolysis-phosphofructokinase, resulting in increased lactate production..$^{29} 30$ Presence of hepatic dysfunction or sepsis may also produce alkalosis in the presence of hyperlactataemia, ${ }^{31}$ although none of our patients had any evidence of liver dysfunction and only three infants had sepsis. Conversely, only in $9 \%$ of instances when arterial $\mathrm{pH}$ was less than 7.20 or in only $12 \%$ of instances of base excess $<-10 \mathrm{mmol} / \mathrm{l}$, was the blood lactate greater than $2.5 \mathrm{mmol} / \mathrm{l}$. The metabolic acidosis in these instances may be related to the accumulation of unmeasured anions not related to lactate metabolism, ${ }^{32}$ the movement of circulating lactate into tissues by acidaemia, ${ }^{33}$ and the limited ability of the preterm kidney to excrete $\mathrm{H}^{+}$and ammonium ions, a lower renal threshold for bicarbonate, and reduced availability in the urine of titratable acid, specifically phosphate. $^{34}$

During anaerobic glycolysis, glucose is metabolised to lactate without any change in the hydrogen ion $\left(\mathrm{H}^{+}\right)$concentration (glucose $+2 \mathrm{ADP}+2 \mathrm{Pi} \rightarrow$ 2lactate $+2 \mathrm{ATP}+2 \mathrm{H}_{2} \mathrm{O}$, where $\mathrm{ADP}$ is adenosine diphosphate, $\mathrm{Pi}$ is inorganic phosphate, and ATP is adenosine triphosphate). Hydrogen ions are produced during hydrolysis of ATP by tissues (ATP + $\mathrm{H}_{2} \mathrm{O} \rightarrow \mathrm{ADP}+\mathrm{Pi}+\mathrm{H}^{+}$). Under anaerobic conditions, tisssues are unable to synthesise ATP from $\mathrm{ADP}$ and $\mathrm{Pi}$, resulting in accumulation of hydrogen ions. Thus protons are generated not though the production of lactate but through the hydrolysis of ATP AT5 $^{36}$ and it is possible to have a selective increase in glycolysis without excessive hydrolysis of ATP. ${ }^{37}$ There is also some evidence that the lactate and hydrogen ions are transported out of muscle at different rates. $^{38}$ Thus lactate ions may be generated without corresponding proton generation.
Mechanisms exist to protect the intracellular $\mathrm{pH}$ such that substantial systemic acidosis produces concomitant inhibition of phosphofructokinase in peripheral glycolytic tissues such as skeletal muscle. ${ }^{29}$ This may mask the hepatic underextraction and clearance of lactate, thereby producing normal blood lactate concentration. Irrespective of the possible underlying mechanism(s) of these discrepancies, it is clear that measurement of base excess cannot be relied on as a proxy measure of blood lactate concentration.

The question then arises, is the measurement of blood lactate of value in sick neonates ? Although our study was not primarily designed to evaluate the prognostic importance of blood lactate concentrations in neonates, we did find an increased inpatient mortality associated with increasing blood lactate concentrations in this selected population. In many instances, blood lactate concentrations rose before any deterioration in the infant's condition had become clinically apparent. Changes in arterial $\mathrm{pH}$ and/or base excess were not informative of the subsequent outcome. In this regard, measurement of blood lactate concentrations, especially serially, may give important prognostic information and an early warning signal. We emphasise the selected nature of our population - that is, only those infants with an indwelling arterial lines were studied.

An early study of the value of blood lactate measurement in RDS showed an inverse relation between blood lactate and the severity of RDS with the lowest lactate values found in infants dying of RDS. ${ }^{9}$ In 1972 Beca and Scopes studied blood lactate and acid-base status in 21 preterm infants with RDS. ${ }^{8}$ Although these babies were relatively more mature and larger than in our study, their findings were strikingly similar to ours. They showed that serial blood lactate measurements were more valuable than a single value, and that babies whose blood lactate concentrations remained low, or fell rapidly, were more likely to survive. Thus in spite of the rapid strides in neonatal intensive care over more than 20 years, the measurement of blood lactate remains a potentially important tool for identifying infants at particularly high risk of death. However, Beca and Scopes claimed that low $\mathrm{pH}$ was a reasonable proxy for hyperlactataemia: our data clearly refute this.

We observed that raised blood lactate concentrations seemed to precede clinical deterioration in six babies, although this was not the aim of our study. Fitzgerald et al, investigating infected preterm babies, found substantially increased blood lactate concentrations in these infants, and suggested that these increases were early markers of sepsis. ${ }^{11}$ The mechanism of hyperlactataemia in sepsis is different to that in RDS, being related to limited oxygen extraction resulting from maldistribution of microcirculatory flow, but these observations emphasise the value of blood lactate concentration as an indicator of a baby's wellbeing under a variety of circumstances. Recently, admission and peak plasma lactate 
concentrations of $15 \mathrm{mmol} / \mathrm{l}$ or greater in near term or term infants referred for ECMO have been shown to predict both mortality ${ }^{10}$ and subsequent neurodevelopmental outcome. ${ }^{39}$ The association between increased lactate and higher mortality in our mainly preterm neonatal population complements these findings.

Recently, normal ranges have been established for capillary blood lactate concentrations in healthy preterm and term infants. ${ }^{4041}$ Furthermore, capillary blood lactate concentrations differ little from arterial concentrations in ill neonates. ${ }^{42}$ This would allow capillary blood samples to be used extensively for blood lactate measurements in ill infants.

Dr Deshpande was supported by the Scientific and Research Committee of Newcastle Health Authority and by the Foundation for the Study of Infant Deaths.

1 Weil $\mathrm{MH}$, Afifi AA. Experimental and clinical studies on lactate and pyruvate as indicators of the severity of acute circulatory failure (shock). Circulation 1970;41:989-1001.

2 Rashkin MC, Bosken C, Bauughman RP. Oxygen delivery in critically ill patients. Relationship to blood lactate and survival. Chest $1985 ; 87 \cdot 580-4$.

3 Cady Jr LD, Weil MH, Afifi AA, Michaels SF, Liu VY, Shubin $\mathrm{H}$. Quantitation of severity of critical illness with special reference to blood lactate. Crit Care Med 1973;1:75-80.

4 Broder G, Weil MH. Excess lactate: an index of reversibility of shock in human patients. Science 1964:143:1457-9.

of shock in human patients. Science 1964;143:1457-9.
Kruse JA, Zaidi SAJ, Carlson RW. Significance of blood lactate levels in critically ill patients with liver disease. $A m \mathcal{F}$ tate levels in critically

6 Peretz DI, Scott HM, Duff J, Dossetor JB, McLean LD, McGregor $M$. The significance of the lacticacidaemia in the shock syndrome. Ann NY Acad Sci 1965;119:1133-41.

7 Vincent JL, Faye PD, Berre J, Leeman M, Degaute JP, Kahn RJ. Serial lactate determinations during circulatory shock. Crit Care Med 1983;11:449-51.

8 Beca JP, Scopes JW. Serial determinations of blood lactate in respiratory distress syndrome. Arch Dis Child 1972;47:550-7.

9 Graven SN, Criscuolo D, Holcomb TM. Blood lactate in the respiratory distress syndrome. Am $\mathcal{F}$ Dis Child 1965;110:614-7.

10 Cheung P-Y, Finer NN. Plasma lactate concentration as a predictor of death in neonates with severe hypoxaemia prequiring extracorporeal membrane oxygenation. f Pediatr 1993;125:763-8.

11 Fitzgerald MJ, Goto M, Myers TF, Zeller WP. Early metabolic effects of sepsis in the preterm infant: Lactic
acidosis and increased glucose requirement. 7 Pediatr acidosis and in

12 Bifano EM. Lactate levels in anaemic preterm infants. Pediatr Res 1991;29:273A

13 Izraeli S, Ben-Sira L, Harell D, Naor N, Ballin A, Davidson $\mathrm{S}$. Lactic acid as a predictor for erythrocyte transfusion in healthy preterm infants with anaemia of prematurity. $\mathcal{F}$ Pediatr 1993;122:629-31.

14 Van Biervliet JP, Van Stekelenburg GJ, Duran M, Wadman SK. Base excess and organic acidaemia. Lancet 1974;ii: $1518-9$.

15 Nimmo GR, Grant IS, Mackenzie SJ. Lactate and acid base changes in the critically ill. Postgrad Med f 1991;67:S56S61.

16 Aduen J, Bernstein WK, Miller J, et al. Relationship between blood lactate concentrations and ionised calcium, glucose and acid-base status in critically ill and noncritically ill patients. Crit Care Med 1995;23:246-52.

17 The International Neonatal Network. The CRIB (Clinical Risk Index for Babies) score: a tool for assessing initial neonatal risk and comparing performance of neonatal intensive care units. Lancet 1992;342:193-8.

18 Harrison J, Hodson AW, Skillen AW, Stappenbeck R, Agius L, Alberti KGMM. Blood glucose, lactate, pyruvate, glycerol, 3-hydroxybutyrate and acetoacetate measurements in man using a centrifugal analyser with a fluorimetric attachment. $\mathcal{F}$ Clin Chem Clin Biochem 1988;26:140-6.

19 Weil MH, Michaels S, Rackow EC. Comparision of blood lactate concentrations in central venous, pulmonary

Murdoch IA, Turner C, Dalton RN. Arterial or mixed venous lactate measurement in critically ill children. Is there a difference? Acta Pediatr Scand 1994;83:412-3.

21 Adams J, Hazard P. Comparision of blood lactate concentrations in arterial and peripheral venous blood (letter). Crit Care Med 1988;16:913-4.

22 Koch G, Wendel H. Adjustment of arterial blood gases and acid base balance in the normal newborn infant during the first week of life. Biol Neonate 1968;12:136-61.

23 Yu J, Payne WW, Ifekwunigwe A, Stevens J. Biochemical status of healthy premature infants in the first 48 hours of life. Arch Dis Child 1965;40:516-25.

24 Aduen J, Bernstein WK, Khastgir T, et al. The use and clinical importance of a substrate-specific electrode for clinical importance of a substrate-specific electrode for 7AMA 1994;272:1678-85.

25 Mizock BA, Falk JL. Lactic acidosis in critical illness. Crit Care Med 1992;20:80-93.

26 Kruse JA, Mehta KC, Carlson RW. Definition of clinically significant lactic acidosis. Chest 1987;92:100S.

27 Sestoft L, Bartels PD. Biochemistry and differential diagnosis of metabolic acidosis. Clin Endocrinol Metab 1983;12:287-302

28 Aduen J, Wiese J, Kerzner R, Altman M, Chernow B. Discordance between increased circulating lactate concentrations and lactic acidosis: results from a large clinical data base $(\mathrm{n}=1,424)$. Crit Care Med 1995;23 (Supp1):A103.

29 Ui M. A role of phosphofructokinase in $\mathrm{pH}$-dependent regulation of glycolysis. Biochim Biophys Acta 1966;124:310-22

30 Relman AS. Metabolic consequences of acid-base disorders. Kidney Int 1972;1:347-59.

31 Record CO, Iles RA, Cohen RD, Williams R. Acid-base and metabolic disturbances in fulminant hepatic failure. Gut 1975;16:144-9.

32 Mecher C, Rackow EC, Astiz ME, et al. Unaccounted for anion in metabolic acidosis during sepsis in humans. Crit Care Med 1991;19:705-11

33 Gutierrez G, Hurtado FJ, Gutierrez M, et al. Net uptake of lactate by rabbit hindlimb during hypoxia. Am Rev Respir Dis 1993;148:1204-9.

$34 \mathrm{McCrory}$ WW. Renal function in the postnatal period. In: McCrory W, ed. Developmental Nephrology. Cambridge: Havard University Press, 1972: 123-61.

35 Alberti KGMM, Cuthbert C. The hydrogen ion in normal metabolism: a review. In: Porter R, Lawrenson G, eds. Metabolic acidosis. Bath: Pitman, 1982: 1-19.

36 Zilva JF. The origin of the acidosis in hyperlactatemia. Ann Clin Biochem 1978;15:40-3.

37 Krebs HA, Woods HF, Alberti KGMM. Hyperlactatemia and lactic acidosis. Essays Med Biochem 1970;1:81-103.

38 Mainwood GW, Worsley-Brown P. The effects of extracellular $\mathrm{pH}$ and buffer concentration on the efflux of lactate from frog sartorius muscle. $\mathcal{F}$ Physiol 1975;250:1-22.

39 Cheung P-Y, Robertson CMT, Finer NN. Plasma lactate as a predictor of early childhood neurodevelopmental outcome of neonates with severe hypoxaemia requiring extracorporeal membrane oxygenation. Arch Dis Child 1996;74:F47-50.

40 Hawdon JM, Ward Platt MP, Aynsley-Green A. Patterns of metabolic adaptation for preterm and term infants in the first neonatal week. Arch Dis Child 1992;67:357-65.

41 Nielsen J, Ytrebo LM, Borud O. Lactate and pyruvate concentrations in capillary blood from newborns. Acta Pediatr Scand 1994;83:920-3.

42 Hawdon JM. Metabolic adaptation and disordered blood glucose homeostasis in the neonate [MD thesis]. University of Newcastle upon Tyne, 1995 